\title{
Radiation Damage Centers in Cholesteryl Heptanoate
}

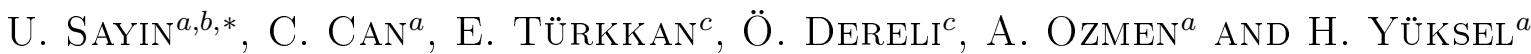 \\ ${ }^{a}$ Physics Department, Selcuk University, Konya, Turkey \\ ${ }^{b}$ Advanced Technology Research and Application Center, Selcuk University, Konya, Turkey \\ ${ }^{c}$ Physics Education Department, Necmettin Erbakan University, Konya, Turkey
}

(Received July 30, 2012; in final form April 6, 2013)

\begin{abstract}
Cholesterol takes part significantly in many biological mechanisms and as important component for manufacture of bile acids, steroid hormones, and several fat-soluble vitamins. To determine magnetic properties of cholesteryl heptanoate $\left(\mathrm{C}_{34} \mathrm{H}_{58} \mathrm{O}_{2}\right)$ which is an important cholesteryl ester in human life and new technology, the single crystals of cholesteryl heptanoate were grown by slow evaporation of concentrated ethyl acetate solution and the grown single crystals were irradiated at room temperature with ${ }^{60} \mathrm{Co} \gamma$ ray. The radical produced by gamma irradiation has been investigated in the range of temperatures $123-330 \mathrm{~K}$ for different orientations of the crystal in a magnetic field by EPR. Radiation damage center was attributed to radical $\mathrm{CH}_{\alpha} \mathrm{CH}_{2 \beta}$. The $g$ factor and hyperfine coupling constants have slight dependence on temperature and evident dependence on the orientation of the magnetic field. Determined $g$ factor and hyperfine coupling constants for the radical $\dot{\mathrm{CH}}_{\alpha} \mathrm{CH}_{2 \beta}$ were found to be anisotropic with the average values $g_{\mathrm{av}}=2.0036,\left(a_{\mathrm{CH}_{\alpha}}\right)_{\mathrm{av}}=14.52 \mathrm{G},\left(a_{\mathrm{CH}_{2 \beta}}\right)_{\mathrm{av}}=25.78 \mathrm{G}$.
\end{abstract}

DOI: $10.12693 /$ APhysPolA.124.70

PACS: $61.80 .-\mathrm{x}$

\section{Introduction}

Cholesterol, shown in Fig. 1a, an important constituent of cell membranes, has a rigid ring system and a short branched hydrocarbon tail.

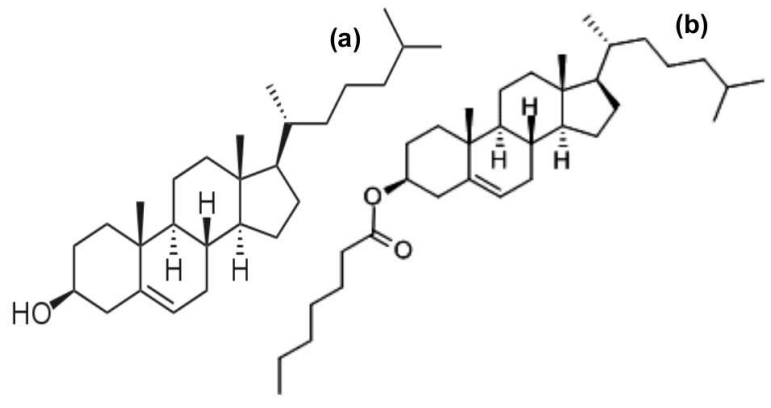

Fig. 1. Molecular structure of (a) cholesterol, (b) cholesteryl heptanoate.

Cholesterol is required to build and maintain membranes; it modulates membrane fluidity over the range of physiological temperatures. Cholesterol reduces the permeability of the plasma membrane to protons (positive hydrogen ions) and sodium ions. Within the cell membrane, cholesterol also functions in intracellular transport, cell signaling and nerve conduction. Within cells, cholesterol is precursor molecule in several biochemical pathways. In the liver, cholesterol is converted to bile, which is then stored in the gallbladder. Cholesterol is an important precursor molecule for the synthesis of vitamin $\mathrm{D}$ and steroid hormones, including the adrenal

\footnotetext{
*corresponding author; e-mail: uakpinar@selcuk.edu.tr
}

gland hormones cortisol and aldosterone as well as sex hormones progesterone, estrogens, and testosterone, and their derivatives.

A cholesteryl ester is, as its name would imply, an ester of cholesterol. The ester bond is formed between the carboxylate group of a fatty acid and the hydroxyl group of cholesterol. Cholesteryl esters have a lower solubility in water than cholesterol and, in other words, are more hydrophobic.

As mentioned above cholesterol and its esters take part significantly in many biological mechanisms and an important component for manufacture of bile acids, steroid hormones, and several fat-soluble vitamins. Numerous experimental investigations have been performed on cholesterol derivative molecules [1-5].

The first report on EPR study of free radicals in X-ray irradiated powdered cholesterol, hormones, and vitamins was done by Rexroad and Gordy and radiation induced radicals were found very stable [6]. Then, gamma irradiated sterol groups were studied at low temperatures [7]. Recently, intense EPR and electron-nuclear double resonance (ENDOR) studies have been undertaken to elucidate the structure of free radicals formed in $\gamma$-irradiated single crystals of selected steroids [8-16].

The studies were prompted by the progress in the technology of radiation sterilization of medical and food products, pharmaceuticals and other materials. In this technology it is essential to have a knowledge of radicals formed upon irradiation.

The aim of our study is to determine the radiation damage to the single crystal cholesteryl heptanoate $\left(\mathrm{C}_{34} \mathrm{H}_{58} \mathrm{O}_{2}\right)$ also called cholesterol enanthate and cholesteryl heptylate, shown in Fig. 1b, which is an important cholesteryl derivative along with the above mentioned chemicals in human life and new technology. 


\section{Experimental}

The white fine crystalline powder of cholesteryl heptanoate $\left(\mathrm{C}_{34} \mathrm{H}_{58} \mathrm{O}_{2}\right)$ was bought from Merck and its single crystals were grown by slow evaporation of concentrated ethyl-acetate solution. The chosen well shaped single crystals of cholesteryl heptanoate were irradiated at room temperature by ${ }^{60} \mathrm{Co} \gamma$ Cell $\mathrm{Px}-\gamma-30$ "ISSLODOVATEL" source of $0.725 \mathrm{kGy} / \mathrm{h}$ for $130 \mathrm{~h}$. The spectra were recorded with a Bruker EMX081 model EPR spectrometer. The low- and high-temperature measurements were carried out using a Bruker temperature control unit between 125-330 K.

\section{Results and discussion}

Since steroids are compounds of great biological importance in nature they became objects of extensive radiation chemistry research, performed both $\mathrm{X}$ - and $\gamma$ irradiated solid phase. Only a few steroids have ever been studied by the EPR method. It is still essential to research the irradiation effects on these steroid groups. The chemical structure of cholesterol and cholesterol derivatives are similar with their 4 ring group. The numbering system of the cholesterol derivatives is shown in Fig. 2, the starting point to the numbering is a carbon atom of $A$ ring. The organic groups of $R^{\prime}$ and $R^{\prime \prime}$ can be changed.

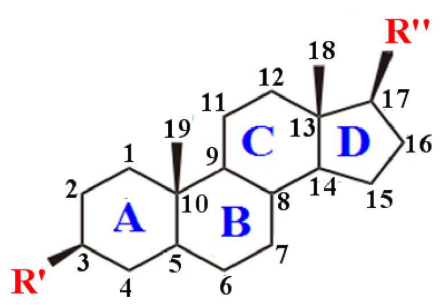

Fig. 2. The numbering scheme of cholesterol derivatives.

Rexroad and Gordy suggested the essential type of resonance pattern for irradiated cholesterol powder, a triplet with doublet substructure arising from three coupling protons in which the two of them had equivalent coupling and the third one had somewhat less coupling than the two equivalent ones. Moreover, Rexroad and Gordy emphasized that determining of the radical structure formed in irradiated cholesterol derivatives is difficult. But they proposed some radical types which can form this type of EPR pattern [6].

According to the EPR spectra, the molecular structure, and the simulations of the spectra it is understood that the unpaired electron seems not to be centered on the side-chain, it is most probably centered on the ring part of the molecule. We proposed that the radical under consideration is formed by removal of a hydrogen atom from $\mathrm{C}(4)$ in $A$ ring (shown in Fig. 2) of the molecule. The unpaired electron is delocalized on carbon atoms $\mathrm{C}(4)$ and $\mathrm{C}(6)$ and interacts with two equivalent protons at $\mathrm{C}(4)$ and $\mathrm{C}(6)$ and another proton attached to $\mathrm{C}(3)$. According to these interactions the radical can be termed as $\dot{\mathrm{C}} \mathrm{H}_{\alpha} \mathrm{CH}_{2 \beta}$ produced in irradiated single crystal of cholesteryl heptanoate. The model structure of proposed radical was given in Fig. 3 .

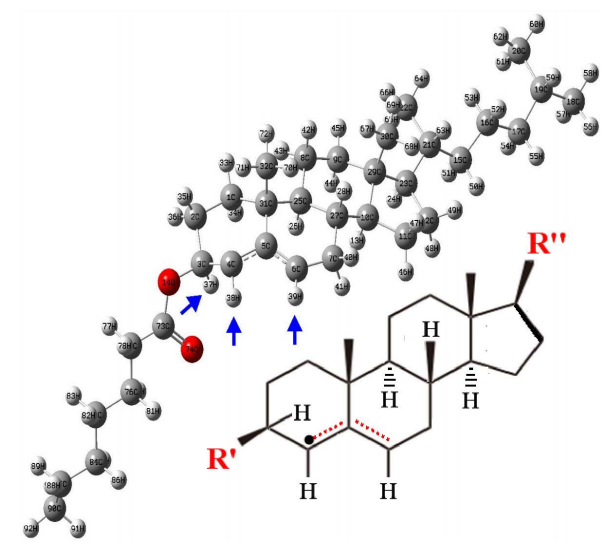

Fig. 3. The schematically radical model structure.

The EPR spectra of cholesteryl heptanoate single crystals were recorded at $10^{\circ}$ intervals in each three $x, y, z$ crystallographic axes in the temperature range 125$330 \mathrm{~K}$. The spectra were found to be slightly dependent on the temperature but significantly dependent upon the orientation of magnetic field shown in Fig. 4 and Fig. 5, respectively.

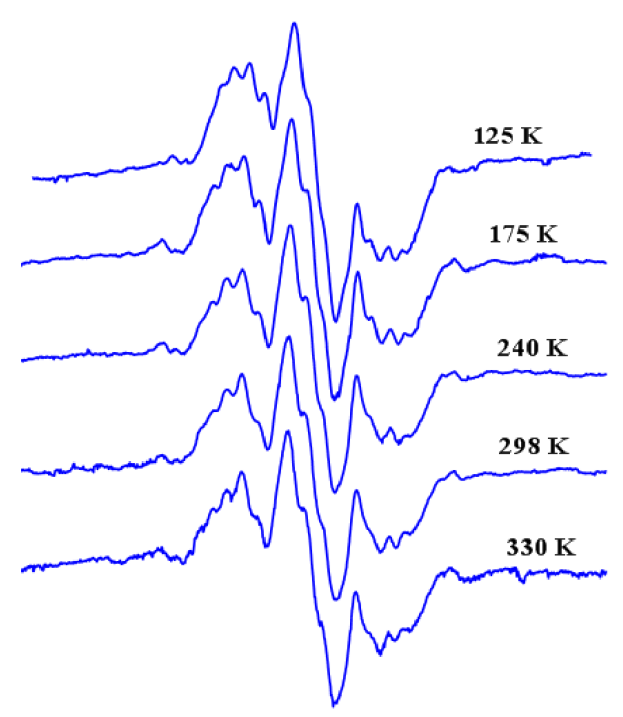

Fig. 4. Temperature dependence of irradiated cholesteryl heptanoate single crystal EPR spectra.

The EPR spectra with the simulation of relevant spectra shown in Fig. 6a and $\mathrm{b}$ have intensity distributions 1:1:2:2:1:1 and 1:3:3:1 of the radical $\dot{\mathrm{C}} \mathrm{H}_{\alpha} \mathrm{CH}_{2 \beta}$, respectively, for different orientations. In Fig. 6a, the hyperfine interaction of unpaired electron with $\alpha$ and $\beta$ hydrogen atoms are magnetically nonequivalent, firstly the 


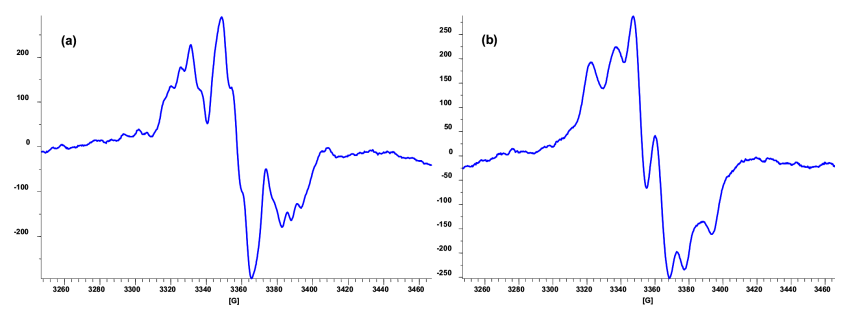

Fig. 5. The EPR spectra of irradiated cholesteryl heptanoate single crystal when the magnetic field is oriented (a) $10^{\circ}$ to the $x$ axis, (b) $50^{\circ}$ to the $y$ axis.

$\mathrm{H}_{2 \beta}$ splitting occur with the intensity ratios $1: 2: 1$, then each peak is split into two (1:1) because of the $\mathrm{H}_{\alpha}$ splitting. In Fig. 6b, the hyperfine coupling constants of $\alpha$ and $\beta$ hydrogen are nearly the same because of the higher anisotropy of $\mathrm{H}_{\alpha}$ coupling, so the intensity ratios of the spectra is 1:3:3:1 which were the ratios of 3 magnetically equivalent hydrogen atoms.

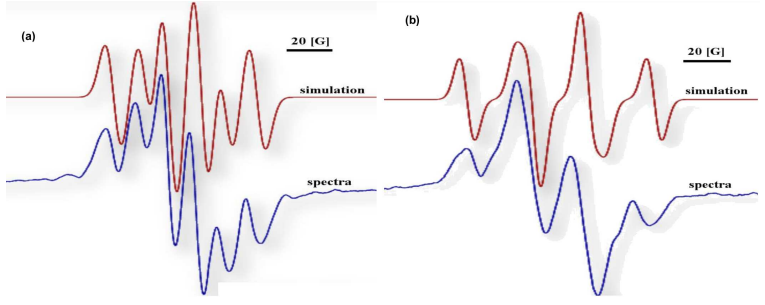

Fig. 6. The EPR spectra and relevant simulation spectra of irradiated cholesteryl heptanoate single crystal when the magnetic field is oriented (a) $160^{\circ}$ to the $z$ axis (b) $100^{\circ}$ to the $x$ axis.

It is known that EPR spectroscopy is one of the most powerful methods for studying the structure and determining the identity of molecules containing unpaired electrons. There are two EPR parameters (the $\boldsymbol{A}$ and $\boldsymbol{g}$ tensors) that contain valuable information about the geometry and electronic structure of the radical systems. The $\boldsymbol{g}$ value provides a fingerprint to identify the paramagnetic centers and important for determining the structure of the radical. The $\boldsymbol{A}$ value of a given nucleus in a radical is highly sensitive to its chemical environment and can be used to determine the spin-density distribution of the radical. The cholesterol derivatives with different side-chain structure have mostly different bonding ring structures in which the produced radical by $\gamma$-irradiation is delocalized and it causes different magnetic environments of unpaired electron at the esters of cholesterol. According to the different bonding ring structures; when the molecule is irradiated, different hydrogen atoms can remove and this causes different $\boldsymbol{g}$-values. Also the hyperfine interaction of the unpaired electron with the protons can change and this causes different $\boldsymbol{A}$-values. This study and the previous EPR studies about cholesterol derivatives show that the produced radical by irradia- tion are delocalized at the different site of ring part of the molecule [6-21]. In other words, the main place and the interaction of the unpaired electron can be different so it is still an important area to determine the produced radicals and the EPR parameters of irradiated cholesterol derivatives.

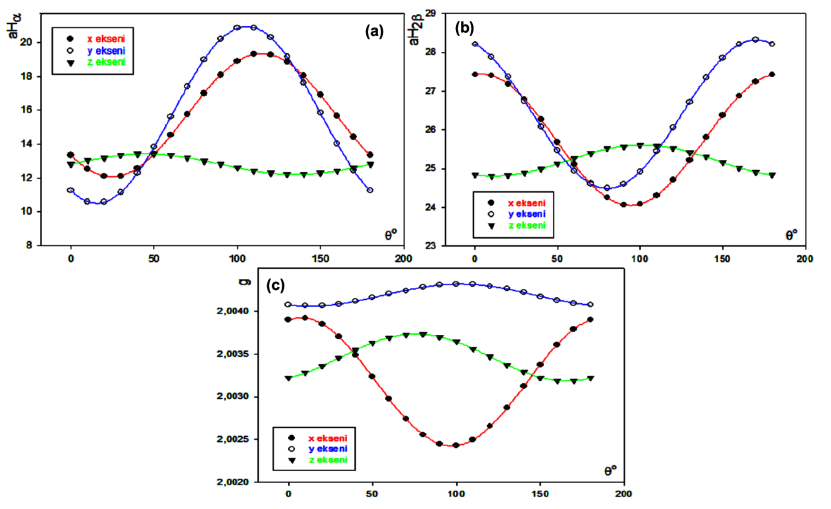

Fig. 7. Angular variation of spectroscopic splitting factor $g$ and hyperfine coupling tensors of $\dot{\mathrm{C}} \mathrm{H}_{\alpha} \mathrm{CH}_{2 \beta}$ radical produced in cholesteryl heptanoate: (a) $A_{\mathrm{H} \alpha}(\theta)$, (b) $A_{\mathrm{H} 2 \beta}(\theta)$, (c) $g(\theta)$.

TABLE

The EPR parameters of $\dot{\mathrm{C}} \mathrm{H}_{\alpha} \mathrm{CH}_{2 \beta}$ radical produced in gamma irradiated cholesteryl heptanoate single crystal.

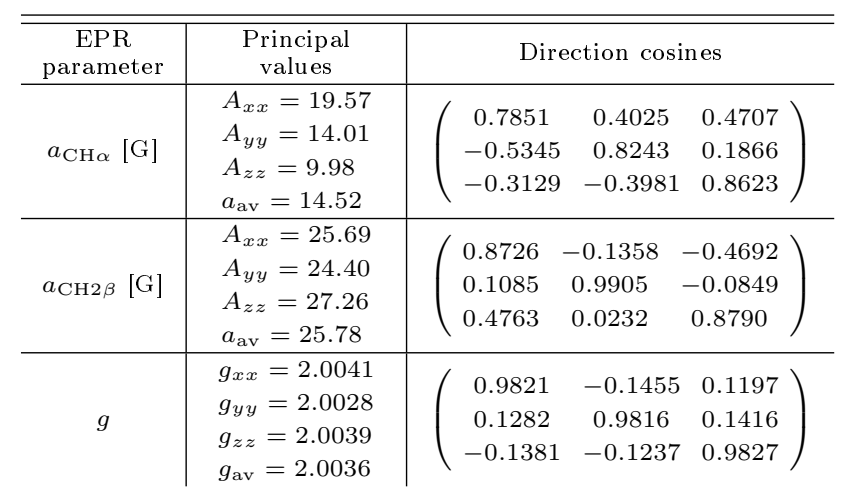

The EPR parameters of detected radical in irradiated cholesteryl heptanoate single crystal is given in Table. The angular variations of the hyperfine interaction tensor, $\boldsymbol{A}(\theta)$ and spectroscopic splitting factor tensor, $\boldsymbol{g}(\theta)$ for the radical $\dot{\mathrm{CH}}_{\alpha} \mathrm{CH}_{2 \beta}$ produced in cholesteryl chloride are shown in Fig. 7.

\section{Conclusion}

In this study, the radiation damage centers produced by gamma irradiation in cholesteryl heptanoate single crystal were determined and the radical structure were proposed for the first time. The EPR parameters of determined stable radical, $\dot{\mathrm{C}} \mathrm{H}_{\alpha} \mathrm{CH}_{2 \beta}$, were obtained and the values were compared with the literature. 
The cholesterol and its derivatives are biologically important molecules so identification of their magnetic properties and the damages of radiation to them are more essential.

\section{Acknowledgments}

This study was financially supported by the BAP, Selcuk University (project number 09401034) and Necmettin Erbakan University (project numbers 121210003 and 121210001).

\section{References}

[1] R. Faiman, K. Larsson, J. Raman. Spectrosc. 5, 3 (1976).

[2] S.C. Goheen, L.J. Lis, J.W. Kauffman, Chem. Phys. Lipids 20, 253 (1977).

[3] S.F. Bush, H. Levin, I.W. Levin, Chem. Phys. Lipids 27, 101 (1980).

[4] D. Di Vizio, K.R. Solomon, M.R. Freeman, Tumori 5, 633 (2008).

[5] E. Ikonen, Nature Rev. Mol. Cell Biol. 9, 125 (2008).

[6] H.N. Rexroad, W. Gordy, Proc. Natl. Acad. Sci. USA 45, 256 (1959).

[7] C.L. Sevilla, D. Becker, M.D. Sevilla, J. Phys. Chem. 90, 2963 (1986).

[8] B. Smaller, M.S. Matheson, J. Chem. Phys. 28, 1169 (1958).
[9] R. Krzyminiewski, A.M. Hafez, A. Szyczewski, J. Pietrzak, J. Mol. Struct. 160, 127 (1987).

[10] R. Krzyminiewski, J. Pietrzak, R. Konopka, J. Mol. Struct. 240, 133 (1990).

[11] A. Szyczewski, K. 'Möbius, J. Mol. Struct. 318, 87 (1994).

[12] A. Szyczewski, Appl. Radiat. Isot. 47, 1675 (1996).

[13] A. Szyczewski, B. Endeward, K. Möbius, Appl. Radiat. Isot. 49, 59 (1998).

[14] B. Çaliskan, E. Aras, B. Aşik, M. Büyüm, M. Birey, Radiat. Eff. Def. 159, 1 (2004).

[15] A. Szyczewski, J. Pietrzak, K. Möbius, Acta Phys. Pol. A 108, 119 (2005).

[16] U. Sayin, H. Yuksel, M. Birey, Radiat. Phys. Chem. 80, 1203 (2011).

[17] R. Krzyminiewski, J. Masiakowski, J. Pietrzak, A. Szyczewski, J. Magn. Res. 46, 300 (1982).

[18] R. Krzyminiewski, A.M. Hafez, J. Pietrzak, A. Szyczewski, J. Magn. Res. 51, 308 (1983).

[19] A.M. Hafez, R. Krzyminiewski, A. Szyczewski, J. Pietrzak, J. Mol. Struct. 130, 301 (1985).

[20] A. Szyczewski, K. Krzyminiewski, A.M. Hafez, J. Pietrzak, Int. J. Radiat. Biol. 50, 841 (1986).

[21] B.T. Yiğit, A. Özmen, Ý. Ercan, M. Eken, M. Birey, Radiat. Eff. Def. 158, 833 (2003). 